\title{
Some ideas for beginners on using a microcomputer in an introductory psychology class
}

\author{
CYNTHIA R. McDANIEL \\ Northern Kentucky University, Highland Heights, Kentucky
}

\begin{abstract}
This tutorial workshop presented several microcomputer applications for a large introductory psychology class with limited equipment. Applications demonstrated were word processing, gradebook management, and demonstrations.
\end{abstract}

A tutorial workshop was presented that explored some of the ways in which a microcomputer could be used in a large introductory psychology class with limited equipment. Demonstrations were provided on ways to use a micro to (1) generate class handouts and examinations (word processing applications), (2) store, manipulate, and report student performance data (gradebook management), and (3) present packaged and homegrown classroom demonstrations (demonstrations).

There is a wide variety of word processing software available, and its application to the classroom is fairly obvious. The advantages of using a word processor multiply when your printer will accept stencils, thus eliminating entirely any transcription phase in producing

The author's mailing address is: Department of Psychology, Northern Kentucky University, Newport, KY 41076. class handouts. Sample handouts were shown, and tips for improving reproduction quality were discussed. Most text publishers are now offering item files on floppy disks for microcomputers. Several of these item files were demonstrated, and their features compared.

Another obvious application of the micro to intro is in storing, manipulating, and reporting the data generated throughout student evaluation. Specialized gradebook programs or adaptations of general data base management programs can be used for this purpose.

Most exciting perhaps is the potential of the micro as an audiovisual aid in demonstrating experiments and psychological phenomena. The micro can become a slide projector or electronic chalkboard to display tables, graphics, lecture outlines, etc. Many traditional pieces of lab equipment can be simulated with the micro, and prepackaged demonstrations are available for doing this. Sources and examples were presented. 